\title{
A summary of drugs targeting enzymes
}

Dr. Rajmohan Seetharaman*, Dr. Manjari Advani**

${ }^{\star}$ Resident doctor, ${ }^{\star *}$ Additional professor

Department of Pharmacology, Lokmanya Tilak Municipal Medical and General Hospital, Mumbai

\section{System-wise list of drugs acting on Enzymes}

\begin{tabular}{|c|c|c|}
\hline Class & Drugs & Mechanism \\
\hline \multicolumn{3}{|c|}{ Autonomic Nervous System } \\
\hline Anticholinesterases & $\begin{array}{l}\text { Physostigmine, } \\
\text { Rivastigmine, Tacrine, } \\
\text { Dyflos, Malathione, } \\
\text { Carbaryl, Propoxur }\end{array}$ & $\begin{array}{l}\text { Acetyl cholinesterase } \\
\text { inhibitors }\end{array}$ \\
\hline $\begin{array}{l}\text { Acetyl cholinesterase } \\
\text { reactivators }\end{array}$ & $\begin{array}{l}\text { Pralidoxime, Obidoxime, } \\
\text { Diacetyl mono-oxime }\end{array}$ & $\begin{array}{l}\text { Acetyl cholinesterase } \\
\text { reactivators }\end{array}$ \\
\hline \multicolumn{3}{|c|}{ Autocoids } \\
\hline NSAIDS & $\begin{array}{l}\text { Aspirin, Ibuprofen, } \\
\text { Nimesulide, Paracetamol }\end{array}$ & $\begin{array}{l}\text { Cyclooxygenase (COX) } \\
\text { inhibitor }\end{array}$ \\
\hline Anti Gout drugs & Allopurinol, Febuxostat & Xanthine Oxidase inhibitor \\
\hline Antimetabolites & Methotrexate & $\begin{array}{l}\text { Dihydro Folate Reductase } \\
\text { inhibitor }\end{array}$ \\
\hline Immunomodulators & Leflunomide & $\begin{array}{l}\text { Dihydro-orotate } \\
\text { dehydrogenase inhibitor }\end{array}$ \\
\hline Immunomodulators & Mycophenolate Mofetil & $\begin{array}{l}\text { Cytosine monophosphate } \\
\text { dehydrogenase inhibitor }\end{array}$ \\
\hline \multicolumn{3}{|c|}{ Renal system } \\
\hline Diuretics & $\begin{array}{l}\text { Acetazolamide, } \\
\text { Dorzolamide, } \\
\text { methazolamide }\end{array}$ & $\begin{array}{l}\text { Carbonic Anhydrase } \\
\text { inhibitors }\end{array}$ \\
\hline $\begin{array}{l}\text { Indirect ET Receptor } \\
\text { antagonists }\end{array}$ & $\begin{array}{l}\text { Phosphoramidon, CGS } \\
35066\end{array}$ & $\begin{array}{l}\text { Endothelin Converting } \\
\text { Enzyme inhibitors }\end{array}$ \\
\hline Antihypertensives & $\begin{array}{l}\text { Captopril, Enalapril, } \\
\text { Lisinopril }\end{array}$ & $\begin{array}{l}\text { Angiotensin Converting } \\
\text { Enzyme Inhibitors }\end{array}$ \\
\hline \multicolumn{3}{|c|}{ Cardiovascular System } \\
\hline Bipyridines & Mirlinone, Cilostazole & $\begin{array}{l}\text { Phosphodiesterase-3- } \\
\text { inhibitor }\end{array}$ \\
\hline
\end{tabular}




\begin{tabular}{|c|c|c|}
\hline Class & Drugs & Mechanism \\
\hline Antiplatelet & Dipyridamole & $\begin{array}{l}\text { Adenosine deaminase } \\
\text { inhibitor } \\
\text { Phosphodiesterase inhibitor }\end{array}$ \\
\hline Cardiac Glycosides & Digoxin, Digitoxin & $\mathrm{Na}+\mathrm{K}+$ ATPase inhibitor \\
\hline Statins & $\begin{array}{l}\text { Atorvastatin, Simvastatin, } \\
\text { Lovastatin }\end{array}$ & $\begin{array}{l}\beta \text {-hydroxy } \beta \text {-methylbutyryl- } \\
\text { CoA (HMG-COA) } \\
\text { Reductase Inhibitor }\end{array}$ \\
\hline Fibrates & $\begin{array}{l}\text { Gemfibrozil, Bezafibrate, } \\
\text { Fenofibrate }\end{array}$ & $\begin{array}{l}\text { Lipoprotien Lipase } \\
\text { Activators }\end{array}$ \\
\hline \multicolumn{3}{|c|}{ Endocrinology } \\
\hline Antiestrogens & Letrozole, Anastrazole & Aromatase inhibitor \\
\hline Antiandrogens & Finasteride, Dutasteride & 5-a-Reductase Inhibitor \\
\hline Erectile dysfunction drugs & Sildenafil, Tadalafil & $\begin{array}{l}\text { Phosphodiesterase-5- } \\
\text { inhibitor }\end{array}$ \\
\hline Antithyroid & PTU, Carbimazole & Thyroid Peroxidase inhibitor \\
\hline Antidiabetics & Insulin & Tyrosine Kinase activator \\
\hline Antidiabetics & Sitagliptin, Saxagliptin & $\begin{array}{l}\text { Dipeptidyl Peptidase-4 } \\
\text { inhibitor }\end{array}$ \\
\hline Antidiabetics & $\begin{array}{l}\text { Acarbose, Miglitol, } \\
\text { Voglibose }\end{array}$ & a-Glucosidase inhibitor \\
\hline Antidiabetics Biguanides & Metformin & $\begin{array}{l}\text { AMP-activated protein } \\
\text { kinase activator }\end{array}$ \\
\hline \multicolumn{3}{|c|}{ Gastrointestinal tract } \\
\hline Proton Pump Inhibitors & $\begin{array}{l}\text { Omeprazole, Pantoprazole, } \\
\text { Lansoprazole, } \\
\text { Dexlansoprazole, } \\
\text { Rabeprazole, Esomeprazole }\end{array}$ & $\mathrm{H}+/ \mathrm{K}+$ ATPase inhibitor \\
\hline \multicolumn{3}{|c|}{ Central nervous system } \\
\hline Antimaniac & Lithium & $\begin{array}{l}\text { Inosine-5'- } \\
\text { monophosphatease } \\
\text { (IMPase) inhibitors }\end{array}$ \\
\hline $\begin{array}{l}\text { Antidepressants } \\
\text { Monoamine oxidase (MAO) } \\
\text { A Inhibitors }\end{array}$ & Moclobemide, Clorgyline & $\begin{array}{l}\text { Monoamine oxidase (MAO) } \\
\text { A inhibitors }\end{array}$ \\
\hline $\begin{array}{l}\text { Antiparkinsonian } \\
\text { Monoamine oxidase (MAO) } \\
\text { B Inhibitors }\end{array}$ & Selegiline & $\begin{array}{l}\text { Monoamine oxidase (MAO) } \\
\text { B inhibitors }\end{array}$ \\
\hline
\end{tabular}




\begin{tabular}{|c|c|c|}
\hline Class & Drugs & Mechanism \\
\hline $\begin{array}{l}\text { Alcohol dehydrogenase } \\
\text { Inhibitors }\end{array}$ & Ethanol & $\begin{array}{l}\text { Alcohol dehydrogenase } \\
\text { inhibitor }\end{array}$ \\
\hline $\begin{array}{l}\text { Alcohol dehydrogenase } \\
\text { Inhibitors }\end{array}$ & Fomepizole & $\begin{array}{l}\text { Alcohol Dehydrogenase } \\
\text { inhibitor }\end{array}$ \\
\hline $\begin{array}{l}\text { Aldehyde Dehydrogenase } \\
\text { Inhibitors }\end{array}$ & Disulfiram & $\begin{array}{l}\text { Aldehyde Dehydrogenase } \\
\text { inhibitor }\end{array}$ \\
\hline Antiepileptic drugs & Valproate & $\begin{array}{l}\text { gamma-Aminobutyric acid } \\
\text { (GABA) Transaminase } \\
\text { inhibitor }\end{array}$ \\
\hline Antiepileptic & Vigabatrin & $\begin{array}{l}\text { gamma-Aminobutyric acid } \\
\text { (GABA) Transaminase } \\
\text { inhibitor }\end{array}$ \\
\hline $\begin{array}{l}\text { Peripheral decarboxylase } \\
\text { Inhibitors }\end{array}$ & Carbidopa & $\begin{array}{l}\text { Dihydroxyphenylalanine } \\
\text { (DOPA) decarboxylase } \\
\text { inhibitor }\end{array}$ \\
\hline $\begin{array}{l}\text { Antiparkinsonian Catechol- } \\
\text { O-methyltransferase } \\
\text { (COMT) Inhibitors }\end{array}$ & Tolcapone, Entacapone & $\begin{array}{l}\text { Catechol-O- } \\
\text { methyltransferase (COMT) } \\
\text { inhibitors }\end{array}$ \\
\hline \multicolumn{3}{|c|}{ Haematology } \\
\hline Parenteral anticoagulants & Heparin & $\begin{array}{l}\text { Antithrombin (AT) Ili } \\
\text { activator }\end{array}$ \\
\hline Coumarin derivatives & Warfarin & $\begin{array}{l}\text { Vitamin K reductase } \\
\text { inhibitors }\end{array}$ \\
\hline Fibrinolytic Drugs & Streptokinase, Urokinase & $\begin{array}{l}\text { Tissue type Plasminogen } \\
\text { activators }\end{array}$ \\
\hline $\begin{array}{l}\text { Platelet Phosphodiesterase } \\
\text { inhibitors }\end{array}$ & Dipyridamol, Cilostazol & $\begin{array}{l}\text { Phosphodiesterase III } \\
\text { inhibitors }\end{array}$ \\
\hline \multicolumn{3}{|c|}{ Antibacterials } \\
\hline$\beta$-lactam antibiotic & Penicillins & $\begin{array}{l}\text { Bacterial Transpeptidase } \\
\text { inhibitors }\end{array}$ \\
\hline$\beta$-Lactamase inhibitors & Clavulanic acid, Sulbactam & $\beta$-Lactamase inhibitors \\
\hline Fluoroquinolones & ciprofloxacin, gemifloxacin & Topoisomerase DNA Gyrase \\
\hline Rifamycins & Rifampicin, Rifabutin & $\begin{array}{l}\text { Prokaryotic RNA } \\
\text { Polymerase inhibitor }\end{array}$ \\
\hline Sulfonamides & $\begin{array}{l}\text { sulfamethoxazole, } \\
\text { Sulfadiazine }\end{array}$ & $\begin{array}{l}\text { Dihydropteroate Synthase } \\
\text { inhibitors }\end{array}$ \\
\hline \multicolumn{3}{|c|}{ Antifungals } \\
\hline
\end{tabular}




\begin{tabular}{|c|c|c|}
\hline Class & Drugs & Mechanism \\
\hline Imidazoles & Ketoconazole, Miconazole & $\begin{array}{l}\text { 14-a-sterol demethylase } \\
\text { inhibitor }\end{array}$ \\
\hline Echinocandins & Caspofungin, Micafungin & $\begin{array}{l}1,3-\beta-d-G l u c a n \text { synthase } \\
\text { inhibitor }\end{array}$ \\
\hline Allylamines & Terbinafine & $\begin{array}{l}\text { Squalene Epoxidase } \\
\text { Inhibitor }\end{array}$ \\
\hline \multicolumn{3}{|c|}{ Antivirals (Non Retroviral) } \\
\hline Anti Herpesvirus agents & Acyclovir, Valacyclovir & $\begin{array}{l}\text { Viral DNA polymerase } \\
\text { inhibitor }\end{array}$ \\
\hline Anti Herpesvirus agents & Ganciclovir, Valganciclovir & $\begin{array}{l}\text { Viral Phosphotransferase } \\
\text { inhibitor }\end{array}$ \\
\hline Antiinfluenza & Oseltamivir, Zanamivir & $\begin{array}{l}\text { Influenza Neuramidase } \\
\text { inhibitor }\end{array}$ \\
\hline Anti Hepatitis B drugs & Entecavir, Adefovir & $\begin{array}{l}\text { Viral DNA polymerase } \\
\text { inhibitor }\end{array}$ \\
\hline Anti Hepatitis C drugs & Sofosbuvir & $\begin{array}{l}\text { HCV RNA Polymerase } \\
\text { inhibitor }\end{array}$ \\
\hline Anti Hepatitis C drugs & Simeprevir & HCV NS3 Protease inhibitor \\
\hline \multicolumn{3}{|c|}{ Antiretrovirals } \\
\hline $\begin{array}{l}\text { Nucleoside Reverse- } \\
\text { transcriptase inhibitors } \\
\text { (NRTI) }\end{array}$ & Zidovudine, Lamivudine & $\begin{array}{l}\text { Viral Reverse Transcriptase } \\
\text { inhibitor }\end{array}$ \\
\hline $\begin{array}{l}\text { Nucleotide Reverse- } \\
\text { transcriptase inhibitors } \\
\text { (NRTI) }\end{array}$ & Tenofovir & $\begin{array}{l}\text { Viral Reverse Transcriptase } \\
\text { inhibitor }\end{array}$ \\
\hline $\begin{array}{l}\text { Non Nucleoside Reverse- } \\
\text { transcriptase inhibitors } \\
\text { (NNRTI) }\end{array}$ & Nevirapine, Efavirenz & $\begin{array}{l}\text { HIV } 1 \text { Reverse Transcriptase } \\
\text { inhibitors }\end{array}$ \\
\hline Protease Inhibitors & $\begin{array}{l}\text { Saquinavir, Ritonavir, } \\
\text { Lopinavir }\end{array}$ & $\begin{array}{l}\text { Virus aspartyl Protease } \\
\text { Inhibitor }\end{array}$ \\
\hline Integrase Inhibitors & Raltegravir, Elvitegravir & HIV encoded Integrase \\
\hline \multicolumn{3}{|c|}{ Antiprotozoal } \\
\hline Antiprotozoal drugs & Eflornithine & $\begin{array}{l}\text { Ornithine decarboxylase } \\
\text { inhibitor }\end{array}$ \\
\hline \multicolumn{3}{|c|}{ Anticancer drugs } \\
\hline Antimetabolites & Methotrexate, Pemetrexed & DHFR inhibitors \\
\hline
\end{tabular}




\begin{tabular}{|c|c|c|}
\hline Class & Drugs & Mechanism \\
\hline Pyrimidine Analogues & 5-FU, Floxuridine & $\begin{array}{l}\text { Thymidylate synthase } \\
\text { inhibitors }\end{array}$ \\
\hline Pyrimidine Analogues & Cytarabine & DNA polymerase inhibitors \\
\hline Antibiotic anticancer drugs & Bleomycin, Dactinomycin & Topoisomerase II inhibitors \\
\hline Epipodophyllotoxins & Etoposide, Teniposide & Topoisomerase II inhibitors \\
\hline Camptothecins & Topotecan, Irinotecan & Topoisomerase I inhibitors \\
\hline Misc. anticancer drugs & Hydroxyurea & Ribonucleotide Reductase \\
\hline Proteosome Inhibitors & Bortezomib, Carfilzonib & Proteosome Inhibitors \\
\hline $\begin{array}{l}\text { Histone Deacetylase } \\
\text { Inhibitors }\end{array}$ & Vorinostat & $\begin{array}{l}\text { Histone Deacetylase } \\
\text { Inhibitors }\end{array}$ \\
\hline \multicolumn{3}{|c|}{ Signalling Pathways } \\
\hline $\begin{array}{l}\text { Monoclonal antibody EGFR } \\
\text { kinase inhibitors }\end{array}$ & Cetuximab, Necitumumab & $\begin{array}{l}\text { epidermal growth factor } \\
\text { receptor (EGFR) Kinase } \\
\text { inhibitors }\end{array}$ \\
\hline $\begin{array}{l}\text { Small molecule EGFR } \\
\text { Kinase Inhibitor }\end{array}$ & Erlotinib, Gefitinib, Afatinib & $\begin{array}{l}\text { epidermal growth factor } \\
\text { receptor (EGFR) Kinase } \\
\text { Inhibitors }\end{array}$ \\
\hline $\begin{array}{l}\text { Small molecule HER2 } \\
\text { Kinase inhibitors }\end{array}$ & Lapatinib, Neratinib & $\begin{array}{l}\text { Human epidermal growth } \\
\text { factor receptor } 2 \text { (HER2) } \\
\text { Kinase inhibitors } \\
\text { epidermal growth factor } \\
\text { receptor (EGFR) inhibitors }\end{array}$ \\
\hline $\begin{array}{l}\text { Monoclonal Antibody HER2 } \\
\text { Kinase inhibitors }\end{array}$ & Trastuzumab, Pertuzumab & $\begin{array}{l}\text { Human epidermal growth } \\
\text { factor receptor } 2 \text { (HER2) } \\
\text { Kinase inhibitors }\end{array}$ \\
\hline $\begin{array}{l}\text { Platelet derived growth } \\
\text { factor inhibitors }\end{array}$ & Olaratumab & $\begin{array}{l}\text { Platelet derived growth } \\
\text { factor inhibitors }\end{array}$ \\
\hline $\begin{array}{l}\text { Mutant B-RAF Kinase } \\
\text { Inhibitors }\end{array}$ & Vemurafenib, Dabrafenib & $\begin{array}{l}\text { Mutant B-RAF Kinase } \\
\text { Inhibitors }\end{array}$ \\
\hline MAP Kinase Inhibitors & Cobimetinib, Trametinib & $\begin{array}{l}\text { mitogen-activated protein } \\
\text { (MAP) Kinase Inhibitors }\end{array}$ \\
\hline $\begin{array}{l}\text { Cyclin dependent kinase } \\
4 / 6 \text { inhibitors }\end{array}$ & Palbociclib, Abemaciclib & $\begin{array}{l}\text { Cyclin dependent kinase } \\
4 / 6 \text { inhibitors }\end{array}$ \\
\hline $\begin{array}{l}\text { Anaplastic lymphoma } \\
\text { kinase inhibitors }\end{array}$ & Alectinib, Ceritinib & $\begin{array}{l}\text { Anaplastic lymphoma } \\
\text { kinase inhibitors }\end{array}$ \\
\hline VEGF inhibitor & $\begin{array}{l}\text { Bevacizumab, } \\
\text { Ramucirumab }\end{array}$ & $\begin{array}{l}\text { Vascular Endothelial Growth } \\
\text { Factor (VEGF) inhibitor }\end{array}$ \\
\hline
\end{tabular}




\begin{tabular}{|c|c|c|}
\hline Class & Drugs & Mechanism \\
\hline $\begin{array}{l}\text { Bruton Tyrosine Kinase } \\
\text { inhibitors }\end{array}$ & Ibrutinib & $\begin{array}{l}\text { Bruton Tyrosine Kinase } \\
\text { inhibitors }\end{array}$ \\
\hline PI3K inhibitors & Idelalisib & $\begin{array}{l}\text { Phosphotidylinositol-4,5- } \\
\text { Biphosphate-3 K inhibitors }\end{array}$ \\
\hline \multicolumn{3}{|c|}{ Immunomodulators } \\
\hline $\begin{array}{l}\text { Lymphocyte signalling } \\
\text { Inhibitors }\end{array}$ & Cyclosporine, Tacrolimus & Calcineurin Inhibitors \\
\hline $\begin{array}{l}\text { Lymphocyte signalling } \\
\text { Inhibitors }\end{array}$ & Sirolimus, Everolimus & $\begin{array}{l}\text { mammalian target of } \\
\text { rapamycin (mTOR) } \\
\text { inhibitors }\end{array}$ \\
\hline Antimetabolites & Mycophenolate Mofetil & $\begin{array}{l}\text { Inosine-5'-monophosphate } \\
\text { (IMP) Dehydrogenase II } \\
\text { Inhibitors }\end{array}$ \\
\hline $\begin{array}{l}\text { Immune cell Adhesion } \\
\text { Inhibitors }\end{array}$ & Natalizumab & a-4-integrins inhibitor \\
\hline
\end{tabular}

\section{Microsomal Enzyme Inducers \& Inhibitors}

\begin{tabular}{|c|c|c|c|}
\hline CYP & Substrates & Inducers & Inhibitors \\
\hline $1 \mathrm{~A} 2$ & $\begin{array}{l}\text { Acetaminophen, } \\
\text { clomipramine, } \\
\text { theophylline, warfarin }\end{array}$ & $\begin{array}{l}\text { Charcoal-broiled foods, } \\
\text { grilled meat, rifampin, } \\
\text { smoking }\end{array}$ & ciprofloxacin \\
\hline $2 A 6$ & $\begin{array}{l}\text { Coumarin, tobacco } \\
\text { nitrosamines, nicotine }\end{array}$ & Efavirenz, phenobarbital & ketoconazole \\
\hline 2B6 & $\begin{array}{l}\text { cyclophosphamide, } \\
\text { ifosfamide, irinotecan }\end{array}$ & $\begin{array}{l}\text { Carbamazepine, } \\
\text { phenobarbital, rifampin }\end{array}$ & $\begin{array}{l}\text { fluoxetine, fluvoxamine, } \\
\text { ketoconazole, }\end{array}$ \\
\hline $2 \mathrm{C9}$ & $\begin{array}{l}\text { phenytoin, tolbutamide, } \\
\text { warfarin }\end{array}$ & $\begin{array}{l}\text { barbiturates, rifabutin, } \\
\text { rifampin }\end{array}$ & $\begin{array}{l}\text { cimetidine, fluconazole, } \\
\text { erythromycin }\end{array}$ \\
\hline 2C19 & $\begin{array}{l}\text { clopidogrel, diazepam, } \\
\text { omeprazole }\end{array}$ & barbiturates, rifampin & fluconazole \\
\hline 2D6 & $\begin{array}{l}\text { chlorpheniramine, } \\
\text { codeine, desipramine, } \\
\text { fluoxetine, fluvoxamine, } \\
\text { haloperidol, imipramine, } \\
\text { morphine, oxycodone } \\
\text { paroxetine, tricyclic } \\
\text { antidepressants }\end{array}$ & Unknown & fluoxetine, quinidine \\
\hline
\end{tabular}




\begin{tabular}{|l|l|l|l|}
\hline CYP & Substrates & Inducers & Inhibitors \\
\hline 2E 1 & $\begin{array}{l}\text { Acetaminophen, } \\
\text { enflurane, ethanol, } \\
\text { halothane }\end{array}$ & Ethanol & disulfiram \\
\hline 3A4 & $\begin{array}{l}\text { amlodipine, astemizole, } \\
\text { cisapride, cyclosporine, } \\
\text { ethinyl estradiol, } \\
\text { nicardipine, nifedipine, } \\
\text { nimodipine, terfinadine }\end{array}$ & $\begin{array}{l}\text { barbiturates, } \\
\text { carbamazepine, } \\
\text { phenytoin, rifampin, St. } \\
\text { John's wort }\end{array}$ & $\begin{array}{l}\text { clarithromycin,, diltiazem, } \\
\text { erythromycin, } \\
\text { fluconazole, grapefruit } \\
\text { juice, ketoconazole, } \\
\text { ritonavir, verapamil }\end{array}$ \\
\hline
\end{tabular}

\section{Newly Approved Drugs acting on enzymes}

\begin{tabular}{|c|c|c|c|}
\hline Generic Name & Date & Mechanism & Treatment \\
\hline upadacitinib & August 16, 2019 & $\begin{array}{l}\text { Janus kinase } 1 \text { (JAK) } \\
\text { inhibitor }\end{array}$ & Rheumatoid Arthritis \\
\hline fedratinib & August 16, 2019 & $\begin{array}{l}\text { highly selective } \\
\text { JAK2 inhibitor }\end{array}$ & Myelofibrosis \\
\hline entrectinib & August 15, 2019 & $\begin{array}{l}\text { selective tyrosine } \\
\text { kinase inhibitor }\end{array}$ & $\begin{array}{l}\text { ROS } 1 \text { positive } \\
\text { NSCLC }\end{array}$ \\
\hline pexidartinib & August 2, 2019 & $\begin{array}{l}\text { tyrosine kinase } \\
\text { inhibitor }\end{array}$ & $\begin{array}{l}\text { Tenosynovial Giant } \\
\text { Cell Tumor }\end{array}$ \\
\hline bevacizumab-bvzr & June 27, 2019 & VEGF inhibitor & $\begin{array}{l}\text { multiple types of } \\
\text { cancer }\end{array}$ \\
\hline alpelisib & May 24, 2019 & kinase inhibitor & $\begin{array}{l}\text { Postmenopausal } \\
\text { women, and men, } \\
\text { with HR positive, } \\
\text { HER2-negative, } \\
\text { breast cancer. }\end{array}$ \\
\hline
\end{tabular}

References

- Brunton L, Knollmann B, Hilal-Dandan R. Goodman \& Gilman's. 13th ed. New York, N.Y.: McGraw-Hill Education LLC.; 2018.

- Katzung B. Basic \& clinical pharmacology. 14th ed. McGraw-Hill Education; 2018.

- Tripathi K. Essentials of medical pharmacology. 7th ed. New Delhi, India: Jaypee Brothers; 2013.

- Sharma H, Sharma K. Sharma \& Sharma's principles of pharmacology. 3rd ed. Hyderabad: Paras Medical Publisher; 2017.

- Murray R. Harper's illustrated biochemistry. 31st ed. New York: McGraw-Hill Medical; 2018.

- Kenakin T. Pharmacology in drug discovery. Amsterdam: Academic Press; 2012, Chapter 6, Enzymes as drug targets, Pages 105-124

- Alexander SP, Fabbro D, Kelly E, Marrion NV, Peters JA, Faccenda E, Harding SD, Pawson AJ, Sharman JL, Southan C, Davies JA. The concise guide to PHARMACOLOGY 2017/18: Enzymes. British journal of pharmacology. 2017 Dec;174:S272-359.

- Ramsay R, Tipton K. Assessment of enzyme inhibition: a review with examples from the development of monoamine oxidase and cholinesterase inhibitory drugs. Molecules. 2017;22(7):1192. 
- New FDA Approved Drugs for 2019 | CenterWatch [Internet]. Centerwatch.com. 2019 [cited 2 September 2019]. Available from: https://www.centerwatch.com/drug-information/fdaapproved-drugs/ 